\title{
The New Form of Mixed Economy with Rationing: Agent-Based Approach
}

\author{
Valery L. Makarov, Albert R. Bakhtizin \\ Central Economics and Mathematics Institute of Russian Academy of Sciences, Moscow, Russian Federation \\ Email: albert.bakhtizin@gmail.com
}

Received 6 December 2013; revised 24 January 2014; accepted 3 February 2014

Copyright @ 2014 by authors and Scientific Research Publishing Inc.

This work is licensed under the Creative Commons Attribution International License (CC BY). http://creativecommons.org/licenses/by/4.0/

(c) (i) Open Access

\begin{abstract}
In the paper, we explore the rationing as a way to implement a variety of incentives for people's activity. We assume that the population is divided into six social clusters with different incentives to work. The first social cluster (business oriented people) has standard incentive: getting profit. Other clusters' members (state and military servants, scientists, art workers, priests, etc.) try to lift on the social stairs, getting higher rank. Having a position of a certain rank, a person obtains the possibility to consume goods according to fixed norms.
\end{abstract}

\section{Keywords}

\section{Social Norms; Agent-Based Models}

\section{Introduction}

There are a sizable number of mixed economy types. Generally speaking, every existing economy is a mixed one in a sense that it uses various economic mechanisms. Every country tries to find optimal combination of the mechanisms. The most famous mechanisms are market, rationing, planning, direct distribution, gifts, and some others.

In the paper, we explore the rationing as a way to implement a variety of incentives for people's activity. We assume that the population is divided into six social clusters with different incentives to work. The first social cluster (business oriented people) has standard incentive: getting profit. Other clusters' members (state and military servants, scientists, art workers, priests, etc.) try to lift on the social stairs, getting higher rank. Having a position of a certain rank, a person obtains the possibility to consume goods according to fixed norms.

So, we have mixture of the two mechanisms: market and rationing. Mathematical economists start to study this kind of models long ago. See, Tobin James [1]. The literature was growing since that time. We indicate a 
few. In Howard David H. [2], one can find some results about dependence of market equilibrium on quantity constraints for a consumer. In Makarov V. L., Vasil'ev V. A. [3], there are numbers of proofs of the equilibrium's existence for various mixed economies.

It is important to mention that in all papers, quantities norms (standards. rates, quotas) are given. The only task related to changing the norms is comparative static's problem. For example, what happened to equilibrium prices? When some norm is increasing?

No studies of mechanisms seek the norms. In reality, the norms are generating by a society. Much depends on the type of a society. Under totalitarian regime the norms are given by a dictator. Other regimes produce a number of ways of how to generate the norms, starting from a decision of relatively small groups (elite) to taking into account the opinion of everybody (referendum).

A number of papers attempt to reveal the mechanism for formation of social standards (see Neumann Martin [4]).

It is clear that mathematical modeling is not an effective method to study the problem. The agent based approach looks promising. There are numbers of papers where the agent based models are successfully used for generating the so called social norms. See, for example, Epstein Joshua M. [5]. Epstein proposes a simple agentbased model where agents learn behavioral rules (i.e., accept certain standards formed in the society). Agents are placed in order in a certain loop. Agents interact with each other. Each agent has a fixed position in the loop and is endowed with two characteristics. The first is "quota", a binary variable which depends on the values of the quotas of the neighboring agents. The neighbors taken into consideration are situated within the radius of vision, which is the second characteristic of the agent. The simulations demonstrated that after certain rules have been established in the society, the majority of agents subconsciously keep following them.

Fent, Thomas described an agent-based model developed for analyzing emergence, stability, and change of social standards within a certain population of artificial agents [6]. The information on social standards is kept within a social network, which unites the agents of the model. Each agent has an inner group (i.e., a set of agents with the same social standards) and an outer group. Accordingly, the agents of the model receive utility from following social rules of their inner group and from refusing the rules of outer group. The model was applied to explain the emergence of temporal social rules, prevalent in certain groups.

The quantities' norms are the special type of social ones. The social norms play roles of constraints in behavior. For example, taboo is a typical social norm. The quantities' norms look as prices. One can buy or not by the price, one can use the quantities' norm or not. Prices can be set by market (people) or by a state agency. So with norms, it can be generated by a society (people) or by an agency.

Essential difference between prices and norms lies on the speed of changes. In the described model below, we consider that the norms change once in a time-unit period.

We can mention the recent paper with some rules of generating the quantities' norms (tips). Savarimuthu et al. described an agent-based model with a micro-level mechanism for the emergence of social standards [7]. The approach differs from the common macro-level mechanism, which is implemented in most papers on social standards. Agents have memory of events and radius of vision, i.e. a parameter which determines the square of the surface where the agent can observe events, related to violating or following social standards. The proposed algorithm for the emergence of social standards is applied to a visit to a restaurant where agents make decisions about tips. Computational simulations revealed that the time for emergence of social standards directly depends on the length of memory (the number of recent events memorized by the agent) and on the value of the radius of vision.

\section{The Model}

We constructed the agent-based model, where agents are people of the six social clusters mentioned above. Moreover people in the clusters are divided to three ranks; high, middle and low ones with exclusion of the first social cluster. The first cluster (business oriented people) has objective to achieve maximal profit. The other people destinations are to get high rank. The quantities' norms associated with the clusters and the ranks in. An agent consumes goods from two sources: from market and rationing spheres. In a simple case we consider that an agent used only one source: market, if he/she belongs to the first cluster, or rationing, otherwise.

Now we describe the first version of the agent-based model, where each social cluster produces one "good", and the norms relate to the first good only. Other "goods" we interpret as development's levels of the clusters. 
So the production function of the first cluster looks as

$$
a_{1}(t)=n_{1}(t)^{\alpha 1(t)} \cdot k_{1}(t-1)^{\beta 1(t)} \cdot a_{2}(t)^{\gamma 2} \cdot a_{3}(t)^{\gamma / 3} \cdot a_{4}(t)^{\gamma 4} \cdot a_{5}(t)^{\gamma 5} \cdot a_{6}(t)^{\gamma 6}
$$

where

$a_{1}(t)$ is a production of the first cluster in the period $t$;

$a_{j}(t)$ is the level of the cluster' $j$ development in the period $t$;

$n_{1}(t)$ is the quantity of agents, populated cluster 1 ;

$k_{1}(t-1)$ is accumulated capital (production funds) to the beginning of the period $t$;

Other clusters' production functions are about the same type. So there is a strong dependence of all clusters on each other. It is impossible to develop one cluster with no development of others.

The consumption of an agent $c_{i}$ is defined by his/her budget, if he belongs to the first cluster and is equal to the existing at the given period norm of a rank \& cluster for others.

So a macro-path $\{a(t), c(t)\}$ of the agent-based model, which is the outcome of simulations, depends on the norms, generated by the mechanism, we try to design.

The comparison of the simulated trajectories can be done by standard way, using well know criteria.

The simple way to generate the norms we used is the following. The initial norms for all clusters and ranks are given. Further agents make influence on the norms according to the formula.

$$
c_{s r}(t)=\left\{\begin{array}{ll}
c_{s r}(t-1) \cdot e_{x(n)} \cdot f_{x(n)}(r) \cdot o_{x(n)}(t-1) ; & s=x \\
c_{s r}(t-1) /\left(e_{x(n)} \cdot f_{x(n)}(r) \cdot o_{x(n)}(t-1) ;\right. & s \neq x
\end{array},\right.
$$

where $x_{x(n)}$-the level of $n$-th agent's influence on the total level of consumption of social cluster $x$-is calculated as:

$$
e_{x(n)}=1+\mu \cdot(1 / n)
$$

with $n$ - the number of agents in the society and $\mu$-a coefficient, which determines the power of the agent. By default $\mu$ equals 10 for agents of the first (high) rank, 3 for the second and 1 for the third rank. This implies that the influence is an order higher for the agents of the first (high) rank if compared to the third rank.

It is important to mention that the state of agents is changing over time. The age of the agent increases and the life expectancy decreases in each moment of time. With a certain probability, estimated on the basis of statistics, the agent can have a child. Upon reaching the age 18, the child with a high probability enters the cluster of parents. Agents can migrate from one cluster to another according to some rules.

\section{Calculations}

We produced a number of scenarios, how agents influence to the norms, divided to the two blocks. The first block deals with assessing the change in the socio-cluster society due to the change in the mechanism for the formation of the norms. The second estimates the impact of various factors on the processes for the formation of the norms.

The first block contains three scenarios to make calculations. The first one is the baseline scenario for the development of the socio-economic system in our model.

The second calculation deals with a slight modification in the work of the model. While the first calculation studied the totality of agents, in which each agent was capable to directly influence the consumption quotas for all social clusters, in the second calculation agents elect representatives of their clusters proportional to the number of agents in the cluster. In other words, we supplement the model with a special (new or additional) cluster. The only function of the agents in this cluster is to regulate social quotas. The agents of the supplementary cluster change quotas according to the formula analogous presented above. The only difference is that the agents of the supplementary cluster have higher level of influence in changing quotas. Moreover, the very change of the quota in each cluster happens stochastically: the quota is changed with probability 1 for the cluster represented by the agent. For other social clusters the quotas are changed with probability 0.5 . This implies that the government elected by the agents fully supports its electorate but does not hurt others strongly.

The third scenario is similar to the second. The difference is higher values of probability for the decrease of 
the quotas for the social clusters, which agents are not represented in the regulating body.

Figure 1 demonstrates the results of calculations with respect to the estimated values of production in all the three scenarios.

We see a justification of the assumption about the need for equal rights of all clusters for successful development of the society. When the regulating body strengthens the policy aimed at discriminating certain social clusters, it is immediately reflected on the volume of production in the whole economic system. Note that a more democratic principle of change of social quotas (when each agent has a direct influence on parameters) is slightly more efficient.

The second block of calculations quantitatively assesses the impact of various factors on the processes for formation of social quotas. Namely, it distinguishes between two mechanisms of formation: by each agent or by a selected group.

First, we studied the dependency of the values for social quotas on the initial number of agents in each social cluster.

In this case the size of the first social cluster was $50 \%$ of the society, and the sizes of other clusters were $10 \%$. In the second case the sizes of all the 6 clusters were equal $(16,(6) \%)$. Note that the initial level of social quotas was the same in both cases. The results for the 8th year of the model are presented in Table 1 (all values are normalized relative to the initial values, i.e., all quotas equal to 1 in the zero year).

The results demonstrate that in the first case (when the first social cluster initially includes $50 \%$ of the society), quotas of the first social cluster increased more than quotas of other clusters. Note that the standard deviation of the values for all clusters is larger than in case of change only by the representatives of a special cluster.

We conducted experiments \#\# 2.1 and 2.2 several times. Each time we obtained a certain (different) set of values for social quotas. Consequently, even making a society more balanced (i.e., with the equal number of agents in social clusters), in the end we inevitably have a differentiation in consumption.

We assessed the dependency of the values of social quotas on the presence/absence of ranks in the society. (With the absence of ranks the agents have equal abilities to change social quotas). Similarly to previous estimations we conducted calculations for 8 model years (see results in Table 2; all values are normalized relative to the initial values, i.e., all quotas equal to 1 in the zero year).

We see that quotas change faster in the presence of ranks. In other words the society with the differentiation $\mathrm{s}$ more inclined to an additional stratification in income.

The final experiment deals with estimating the dependency of social quotas on their initial values. In initialize-

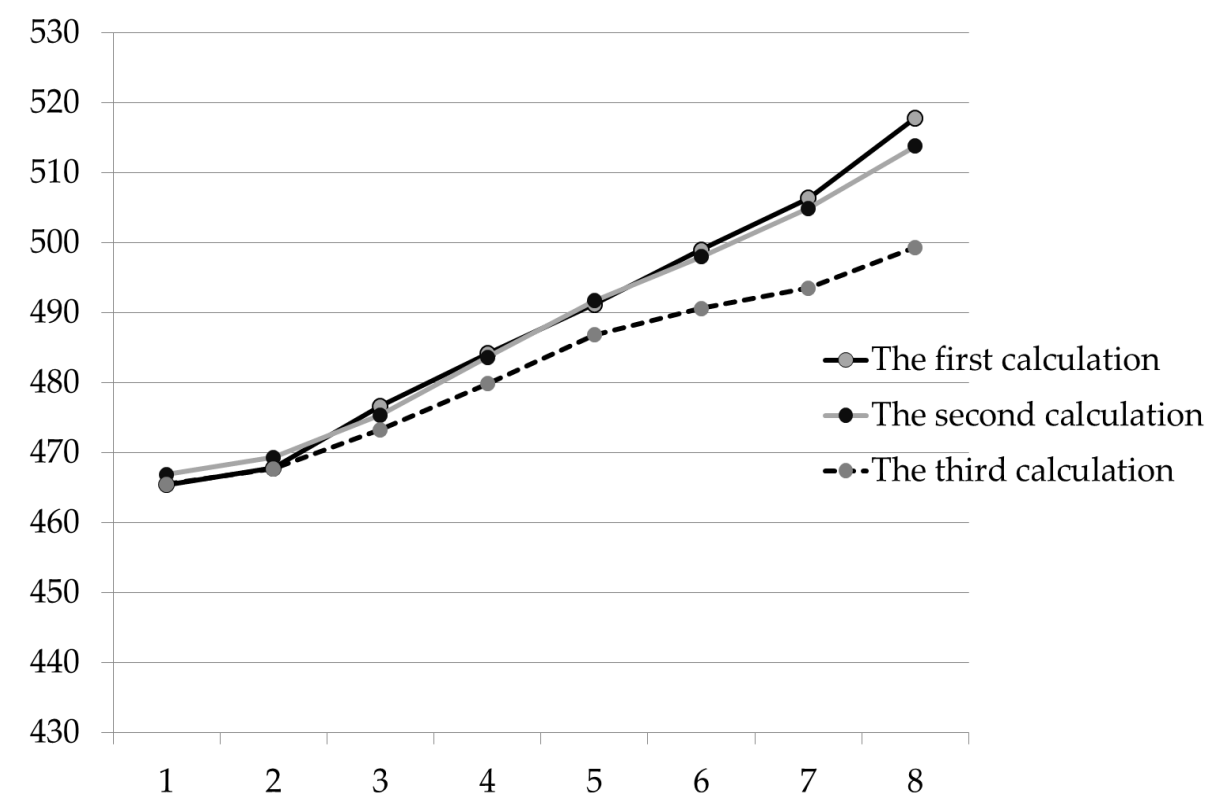

Figure 1. The results of computational simulations ( $\mathrm{X}$ coordinates is years, $\mathrm{Y}$ coordinates is the volume of production). 
Table 1. The impact of the initial number of agents in clusters.

\begin{tabular}{ccccc}
\hline Social cluster & $\begin{array}{c}\text { Experiment 1.1 } \\
\text { (each agent can } \\
\text { change quota) }\end{array}$ & $\begin{array}{c}\text { Experiment 1.2 } \\
\text { (quotas are changed only by } \\
\text { representatives of a special cluster) }\end{array}$ & $\begin{array}{c}\text { Experiment 2.1 } \\
\text { (each agent can } \\
\text { change quota) }\end{array}$ & $\begin{array}{c}\text { Experiment 2.2 } \\
\text { (quotas are changed only by } \\
\text { representatives of a special cluster) }\end{array}$ \\
\hline First & 1.053 & 1.042 & 1.001 & 0.996 \\
Second & 0.962 & 0.944 & 1.011 & 1.002 \\
Third & 0.930 & 0.981 & 0.983 & 1.010 \\
Fourth & 0.954 & 0.956 & 1.035 & 0.987 \\
Fifth & 0.936 & 0.948 & 0.996 & 1.003 \\
Sixth & 0.933 & 0.967 & 1.022 & 0.989 \\
$\begin{array}{c}\text { Standard deviation } \\
\text { (for all clusters) }\end{array}$ & 0.03060 & 0.02568 & 0.01479 & 0.00713 \\
\hline
\end{tabular}

Table 2. The impact of the number of ranks.

\begin{tabular}{ccc}
\hline Social cluster & Ranks are present & Ranks are absent \\
\hline First & 0.981 & 0.995 \\
Second & 0.943 & 0.981 \\
Third & 1.034 & 0.975 \\
Fourth & 0.967 & 1.036 \\
Fifth & 1.045 & 0.983 \\
Sixth & 0.954 & 1.055 \\
Standard deviation (for all clusters) & 0.03478 & 0.02759 \\
\hline
\end{tabular}

ing the model we increased the quotas for the first cluster, decreased for the sixth cluster, and left unchanged for other clusters. Our purpose was to study the impact of initial misbalance in social quotas on the values of social quotas in the long run (15 years).

The results (Table 3) demonstrate a clear long run tendency: the discrepancy in social quotas increases. This implies that no movement towards balance can happen without an exogenous disturbance.

To sum up, the results of computational simulations revealed that:

1) Successful development of a society with social clusters requires equal rights of social clusters;

2) Democratic principle of change in quotas (when each individual has a direct influence on the values of quotas) is a more efficient economic mechanism;

3) Social quotas change faster for the dominant social cluster than for other clusters;

4) Even trying to make a society more balanced (i.e. with equal number of individuals in social clusters) at the end we inevitably face differentiation in production and consumption;

5) A society with differentiation is more inclined to additional stratification in the level of consumption.

\section{Conclusions}

So, in this paper, we explore the rationing as a way to implement a variety of incentives for people's activity. We constructed the agent-based model, where agents are people of the six social clusters. The first social cluster (business oriented people) has standard incentive: getting profit. Other clusters' members (state and military servants, scientists, art workers, priests, etc.) try to lift on the social stairs, getting higher rank. We produced a number of scenarios on how agents influence the norms, and divided them to two blocks. The first block deals with assessing the change in the socio-cluster society due to the change in the mechanism for the formation of the norms. The second estimates the impact of various factors on the processes for the formation of the norms.

In near future, we plan coming closer to reality in three ways:

1) Introduction of spatial and regional feature of the model.

2) More specification of the agents.

3) Comparison of mechanisms for changing norms related the different decision making, going from a political system. 
Table 3. The impact initial values of social quotas.

\begin{tabular}{cccc}
\hline Social cluster & Initial values of social quotas & Each agent changes quotas & $\begin{array}{c}\text { Only representatives of a } \\
\text { special cluster change quotas }\end{array}$ \\
\hline First & 1.500 & 1.544 & 1.525 \\
Second & 1.000 & 0.820 & 0.833 \\
Third & 1.000 & 0.811 & 0.824 \\
Fourth & 1.000 & 0.810 & 0.829 \\
Fifth & 1.000 & 0.809 & 0.826 \\
Sixth & 0.500 & 0.397 & 0.406 \\
\hline
\end{tabular}

\section{Acknowledgements}

This work is supported by Russian Humanitarian Scientific Fund (grants No. 14-02-00431 and No. 12-0200082).

\section{References}

[1] Tobin, J. (1952) A Survey of the Theory of Rationing. Econometrica, 20, 1952, 521-553. http://dx.doi.org/10.2307/1907642

[2] Howard, D.H. (1977) Rationing, Quantity Constraints, and Consumption Theory. Econometrica, 45, 399-412. http://dx.doi.org/10.2307/1911217

[3] Makarov, V.L. and Vasil'ev, V.A. (1989) Equilibrium, Rationing an Stability. Matecon, 25, 4-95.

[4] Neumann, M. (2010) Norm Internalisation in Human and Artificial Intelligence. Journal of Artificial Societies and Social Simulation, 13, 2010.

[5] Epstein, J.M. (2000) Learning to be Thoughtless: Social Norms and Individual Computation. The Brookings Institution and Santa Fe Institute, Center on Social and Economic Dynamics, Working Paper No. 6, 2000.

[6] Fent, T. (2006) Collective Social Dynamics and Social Norms. MPRA Paper No. 2841.

[7] Bastin, T.R.S., Stephen, C., Maryam, A.P. and Martin, K.P. (2010) Obligation Norm Identification in Agent Societies. Journal of Artificial Societies and Social Simulation, 13. http://jasss.soc.surrey.ac.uk/13/4/3.html 